Mathematics in Applied Sciences and Engineering

Volume 2, Number 1, March 2021, pp.22-31

https://ojs.lib.uwo.ca/mase

https://doi.org/10.5206/mase/13393

\title{
TRAVELING WAVES IN COOPERATIVE PREDATION: RELAXATION OF SUBLINEARITY
}

\author{
SRIJANA GHIMIRE AND XIANG-SHENG WANG
}

\begin{abstract}
In this paper, we investigate traveling wave solutions of a diffusive predator-prey model which takes into consideration hunting cooperation. The sublinearity condition is violated for the function of cooperative predation. When the basic reproduction number for the diffusion-free model is greater than one, we find a critical wave speed below which no positive traveling wave solution shall exist. On the other hand, if the wave speed exceeds this critical value, we prove the existence of a positive traveling wave solution connecting the predator-free equilibrium to the unique positive equilibrium under a technical assumption of weak cooperative predation. The key idea of the proof contains two major steps: (i) we construct a suitable pentahedron and find inside it a trajectory connecting the predator-free equilibrium; and (ii) we construct a suitable Lyapunov function and use LaSalle invariance principle to prove that the trajectory also connects the positive equilibrium. At the end of this paper, we propose five open problems related to traveling wave solutions in cooperative predation.
\end{abstract}

\section{Introduction}

The Lotka-Volterra system has been widely used in the models of predation ever since Lotka and Volterra did two independent studies $[17,20]$ near one century ago. Taking spatial diffusion into consideration, a general diffusive Lotka-Volterra system can be written as

$$
\begin{aligned}
& \partial_{t} u=d_{1} \partial_{x x} u+b(u)-f(u, v), \\
& \partial_{t} v=d_{2} \partial_{x x} v+r f(u, v)-\mu v,
\end{aligned}
$$

where $u(t, x)$ and $v(t, x)$ correspond to the densities of prey and predator, respectively. The constants $d_{1}$ and $d_{2}$ are two non-negative diffusion rates. In the absence of the predator, the dynamics of prey is determined by the function $b(u)$, which usually possesses a stable positive equilibrium $K$. The constant $K$ is also referred to as the carrying capacity in the literature. The only nonlinearity in the model system is the predation rate $f(u, v)$ which is an increasing function of both $u$ and $v$, and $f(0, v)=f(u, 0)=0$. The constants $r$ and $\mu$ are rates of energy conversation and predator death, respectively. It is obvious that the model system has a predator-free equilibrium $(K, 0)$. We also assume that it has a positive equilibrium $(U, V)$, where $V=r b(U) / \mu$ and $U$ is a positive root of $b(U)-f(U, r b(U) / \mu)=0$. A traveling wave solution connecting the predator-free equilibrium to the positive equilibrium is a solution of the special form $(u(x+c t), v(x+c t))$ with $c>0$ such that $(u(-\infty), v(-\infty))=(K, 0)$ and $(u(\infty), v(\infty))=(U, V)$. There are plenty of results on the existence

Received by the editors 6 December 2020; accepted 29 January 2021; published online 1 February 2021.

2000 Mathematics Subject Classification. Primary 92D25; Secondary 35K57, 34B40.

Key words and phrases. Predator-prey model; cooperative predation; traveling waves; sublinearity. 
theory of traveling wave solutions to the Lotka-Volterra system of predation; see [5, 10, 11, 12, 13, 16, 22] and references therein. In the aforementioned literature, sublinearity of the predation rate is a crucial condition to prove existence of traveling wave solutions; that is, one should assume that there exists $M>0$ such that

$$
f(u, v) \leq M v
$$

for all $u \in[0, K]$ and $v \geq 0$. The objective of this paper is to relax this sublinearity condition and establish existence theory of travelling waves for a diffusive model of cooperative predation.

Cooperative hunting plays an important role in Phylogenetics [2] and is necessary for some predators [19]. For example, the Yellowstone wolves of larger group size have bigger success rates in capturing bison, their most formidable prey [18]. During the season of nonbreeding, Harris' hawks in New Mexico will improve capture success by cooperative hunting [3]. D. discoideum, which is a soil amoeba feeding on bacteria, lives as single cells during most of the time, but will develop social cooperation if it is under starvation conditions $[4,15]$. A two-dimensional Lotka-Volterra system was proposed in [1] which considers logistic growth of the prey and cooperative hunting on the predator. This model was extended in [14] to investigate Allee effects on the prey. In this paper, we will introduce spatial diffusion to the predator and study the following diffusive predator-prey model with cooperative predation.

$$
\begin{aligned}
& \partial_{t} u=\alpha-\delta u-p u v-q u v^{2}, \\
& \partial_{t} v=d \partial_{x x} v+r\left(p u v+q u v^{2}\right)-\mu v,
\end{aligned}
$$

where $\alpha$ and $\delta$ are the constant birth rate and per capita death rate of the prey. The nonlinear predation rate is composed of mass-action predation puv and cooperative predation $q u v^{2}$. For simplicity, we neglect the spatial diffusion of the prey and assume that the predator has a constant diffusion rate $d>0$. The conversation rate of predation energy is $r>0$ and the per capita death rate of the predator is $\mu>0$.

It is obvious that the model system has a predator-free equilibrium $(K, 0)$, where $K=\alpha / \delta$ is the carrying capacity of the prey. Define

$$
R_{0}=\frac{r p K}{\mu}=\frac{r p \alpha}{\mu \delta}
$$

as the basic reproduction number for the diffusion-free ordinary differential system. Throughout this paper, we will assume that $R_{0}>1$. A simple argument shows that the model system possesses a unique positive equilibrium $(U, V)$ with

$$
\alpha-\delta U=p U V+q U V^{2}=\mu V / r .
$$

We are interested in the traveling wave solution $(u(x+c t), v(x+c t))$ that connects the predator-free equilibrium $(K, 0)$ to the positive equilibrium $(U, V)$. As we mentioned earlier, the nonlinear predation rate $f(u, v)=p u v+q u v^{2}$ does not satisfy the sublinearity condition (1.3) and the results in the existing literature do not apply. To overcome this difficulty, we will modify the shooting method which was introduced by Dunbar [6, 7], and later developed by Hosono and Ilyas [8, 9] and by Huang [12, 13]. Especially, we will apply an extension of the technique introduced by Huang $[12,13]$ to prove the existence of a positive traveling wave connecting the predator-prey equilibrium.

The rest of this paper is organized as follows. In Section 2, we introduce non-dimensional scales for system (1.4)-(1.5) and state our main theorem. In Section 3, we convert the traveling wave equations to a three-dimensional dynamical system and construct a suitable pentahedron. In Section 4, we analyze 
the unstable manifold of the predator-free equilibrium and find a trajectory which approaches the predator-free equilibrium as $\xi \rightarrow-\infty$ and stays in the pentahedron for all $\xi \in \mathbb{R}$. In Section 5 , we use Lyapunov function technique to prove that this trajectory converges to the positive equilibrium as $\xi \rightarrow \infty$. In Section 6, we give a proof of our main theorem. In Section 7, we conclude our paper with a brief discussion and propose some open problems.

\section{NONDIMENSIONALIZATION AND MAIN THEOREM}

For convenience, we nondimensionalize the model by scaling the variables:

$$
\tilde{t}=\delta t, \tilde{x}=\frac{x}{\sqrt{d / \delta}}, \tilde{u}=\frac{u}{K}, \tilde{v}=\frac{v}{r K} .
$$

Recall that $K=\alpha / \delta$. The system (1.4)-(1.5) is rewritten as

$$
\begin{aligned}
& \partial_{\tilde{t}} \tilde{u}=1-\tilde{u}-\tilde{p} \tilde{u} \tilde{v}-\tilde{q} \tilde{u} \tilde{v}^{2}, \\
& \partial_{\tilde{t}} \tilde{v}=\partial_{\tilde{x} \tilde{x}} \tilde{v}+\tilde{p} \tilde{u} \tilde{v}+\tilde{q} \tilde{u} \tilde{v}^{2}-\tilde{\mu} \tilde{v},
\end{aligned}
$$

where

$$
\tilde{p}=\frac{p r K}{\delta}, \tilde{q}=\frac{q r^{2} K^{2}}{\delta}, \tilde{\mu}=\frac{\mu}{\delta} .
$$

Hence, we may assume without loss of generality that $\alpha=\delta=d=r=1$ and drop the tilde in the above system. Denote $\xi=x+$ ct. The traveling wave solution is a solution to the boundary value problem

$$
\begin{aligned}
& c u^{\prime}=1-u-p u v-q u v^{2}, \\
& c v^{\prime}=v^{\prime \prime}+p u v+q u v^{2}-\mu v,
\end{aligned}
$$

together with the boundary conditions

$$
u(-\infty)=1, v(-\infty)=0, u(\infty)=U, v(\infty)=V,
$$

where

$$
1-U=p U V+q U V^{2}=\mu V .
$$

The condition $R_{0}>1$ is simplified as $p>\mu$. Our main theorem is stated as below.

Theorem 2.1. Let $\alpha=\delta=d=r=1$. If $p>\mu$, then for any $c>2 \sqrt{p-\mu}$, there exists $Q>0$ such that the boundary value problem (2.5)-(2.7) with $q \in[0, Q]$ has a positive solution which corresponds to a traveling wave solution to (1.4)-(1.5) connecting the predator-free equilibrium to the positive equilibrium. On the other hand, if $p>\mu$ and $0 \leq c<2 \sqrt{p-\mu}$, then the boundary value problem (2.5)-(2.7) with $q \geq 0$ does not have a positive solution.

\section{Dynamical System APPROACH}

We convert the boundary value problem (2.5)-(2.6) into a three-dimensional dynamical system

$$
\begin{aligned}
c u^{\prime} & =1-u-p u v-q u v^{2}, \\
v^{\prime} & =c v-c w, \\
c w^{\prime} & =p u v+q u v^{2}-\mu v .
\end{aligned}
$$


We intend to find a trajectory such that the boundary conditions (2.7) are satisfied. Let $\varepsilon>0$ be a positive constant to be determined later. We obtain from the above equations

$$
(c u+\varepsilon v+c w)^{\prime}=1-\varepsilon(c u+\varepsilon v+c w)-(1-c \varepsilon) u-\left(\mu-c \varepsilon-\varepsilon^{2}\right) v .
$$

We will choose $\varepsilon$ to be sufficiently small so that

$$
1-c \varepsilon>0, \mu-c \varepsilon-\varepsilon^{2}>0
$$

namely, we require

$$
\varepsilon<\min \left\{\frac{1}{c}, \frac{c+\sqrt{c^{2}+4 \mu}}{2}\right\} .
$$

Let $0<k_{1}<k_{2}$ be two positive constants to be determined later. We introduce a pentahedron $P$ with two parallel triangular bases

$$
\begin{aligned}
& B_{0}:=\left\{u=0, k_{1} v<w<k_{2} v, c u+\varepsilon v+c w<1 / \varepsilon\right\}, \\
& B_{1}:=\left\{u=1, k_{1} v<w<k_{2} v, c u+\varepsilon v+c w<1 / \varepsilon\right\},
\end{aligned}
$$

and three trapezoid sides

$$
\begin{aligned}
& S_{0}:=\left\{c u+\varepsilon v+c w=1 / \varepsilon, 0<u<1, k_{1} v<w<k_{2} v\right\}, \\
& S_{1}:=\left\{w=k_{1} v, 0<u<1, c u+\varepsilon v+c w<1 / \varepsilon\right\}, \\
& S_{2}:=\left\{w=k_{2} v, 0<u<1, c u+\varepsilon v+c w<1 / \varepsilon\right\} .
\end{aligned}
$$

We have the following lemma.

Lemma 3.1. Assume $p>\mu, c>2 \sqrt{p-\mu}$ and $q<\varepsilon^{2}\left[c^{2} / 4-(p-\mu)\right]$. Define

$$
k_{1}:=\frac{1-\sqrt{1-4\left(p+q / \varepsilon^{2}-\mu\right) / c^{2}}}{2}, k_{2}:=\frac{1+\sqrt{1+4 \mu / c^{2}}}{2} .
$$

The direction fields of the ordinary differential system (3.1)-(3.3) point inward on $B_{0} \cup B_{1} \cup S_{0}$ and outward on $S_{1} \cup S_{2}$. In other words, if $\left(u\left(\xi_{1}\right), v\left(\xi_{1}\right), w\left(\xi_{1}\right)\right) \in B_{0} \cup B_{1} \cup S_{0}$ for some $\xi_{1} \in \mathbb{R}$, then there exists $\delta>0$ such that $(u(\xi), v(\xi), w(\xi)) \in P$ for all $\xi \in\left(\xi_{1}, \xi_{1}+\delta\right)$ and $(u(\xi), v(\xi), w(\xi)) \notin P$ for all $\xi \in\left(\xi_{1}-\delta, \xi_{1}\right)$; and if $\left(u\left(\xi_{1}\right), v\left(\xi_{1}\right), w\left(\xi_{1}\right)\right) \in S_{1} \cup S_{2}$ for some $\xi_{1} \in \mathbb{R}$, then there exists $\delta>0$ such that $(u(\xi), v(\xi), w(\xi)) \notin P$ for all $\xi \in\left(\xi_{1}, \xi_{1}+\delta\right)$ and $(u(\xi), v(\xi), w(\xi)) \in P$ for all $\xi \in\left(\xi_{1}-\delta, \xi_{1}\right)$.

Proof. It is obvious that $0 \leq u \leq 1,0 \leq v \leq 1 / \varepsilon^{2}$ and $0 \leq w \leq 1 /(c \varepsilon)$ for any $(u, v, w) \in P$.

If $\left(u\left(\xi_{1}\right), v\left(\xi_{1}\right), w\left(\xi_{1}\right)\right) \in B_{0}$ for some $\xi_{1} \in \mathbb{R}$, then $u\left(\xi_{1}\right)=0$. It follows from (3.1) that $u^{\prime}\left(\xi_{1}\right)=$ $1 / c>0$. Hence, there exists $\delta>0$ such that $u(\xi)>0$ for $\xi \in\left(\xi_{1}, \xi_{1}+\delta\right)$ and $u(\xi)<0$ for $\xi \in\left(\xi_{1}-\delta, \xi_{1}\right)$. This implies that the direction field on $B_{0}$ is inward.

If $\left(u\left(\xi_{1}\right), v\left(\xi_{1}\right), w\left(\xi_{1}\right)\right) \in B_{1}$ for some $\xi_{1} \in \mathbb{R}$, then $u\left(\xi_{1}\right)=1$. It follows from (3.1) that $u^{\prime}\left(\xi_{1}\right)=$ $-\left[p v\left(\xi_{1}\right)+q v^{2}\left(\xi_{1}\right)\right] / c<0$. Hence, there exists $\delta>0$ such that $u(\xi)<1$ for $\xi \in\left(\xi_{1}, \xi_{1}+\delta\right)$ and $u(\xi)>1$ for $\xi \in\left(\xi_{1}-\delta, \xi_{1}\right)$. This implies that the direction field on $B_{1}$ is inward.

If $\left(u\left(\xi_{1}\right), v\left(\xi_{1}\right), w\left(\xi_{1}\right)\right) \in S_{0}$ for some $\xi_{1} \in \mathbb{R}$, then $c u\left(\xi_{1}\right)+\varepsilon v\left(\xi_{1}\right)+c w\left(\xi_{1}\right)=1 / \varepsilon$. It follows from (3.4) that $c u^{\prime}\left(\xi_{1}\right)+\varepsilon v^{\prime}\left(\xi_{1}\right)+c w^{\prime}\left(\xi_{1}\right)=-(1-c \varepsilon) u\left(\xi_{1}\right)-\left(\mu-c \varepsilon-\varepsilon^{2}\right) v\left(\xi_{1}\right)<0$. Hence, there exists $\delta>0$ such that $c u(\xi)+\varepsilon v(\xi)+c w(\xi)<1 / \varepsilon$ for $\xi \in\left(\xi_{1}, \xi_{1}+\delta\right)$ and $c u(\xi)+\varepsilon v(\xi)+c w(\xi)>1 / \varepsilon$ for $\xi \in\left(\xi_{1}-\delta, \xi_{1}\right)$. This implies that the direction field on $S_{0}$ is inward. 
If $\left(u\left(\xi_{1}\right), v\left(\xi_{1}\right), w\left(\xi_{1}\right)\right) \in S_{1}$ for some $\xi_{1} \in \mathbb{R}$, then $w\left(\xi_{1}\right)=k_{1} v\left(\xi_{1}\right)$. It follows from (3.2) and (3.3) that

$$
\frac{w^{\prime}\left(\xi_{1}\right)-k_{1} v^{\prime}\left(\xi_{1}\right)}{c}<\frac{p+q / \varepsilon^{2}-\mu}{c^{2}} v\left(\xi_{1}\right)-k_{1} v\left(\xi_{1}\right)+k_{1}^{2} v\left(\xi_{1}\right)=0 .
$$

Hence, there exists $\delta>0$ such that $w(\xi)<k_{1} v(\xi)$ for $\xi \in\left(\xi_{1}, \xi_{1}+\delta\right)$ and $w(\xi)>k_{1} v(\xi)$ for $\xi \in\left(\xi_{1}-\delta, \xi_{1}\right)$. This implies that the direction field on $S_{1}$ is outward.

If $\left(u\left(\xi_{1}\right), v\left(\xi_{1}\right), w\left(\xi_{1}\right)\right) \in S_{2}$ for some $\xi_{1} \in \mathbb{R}$, then $w\left(\xi_{1}\right)=k_{2} v\left(\xi_{1}\right)$. It follows from (3.2) and (3.3) that

$$
\frac{w^{\prime}\left(\xi_{1}\right)-k_{2} v^{\prime}\left(\xi_{1}\right)}{c}>-\frac{\mu}{c^{2}} v\left(\xi_{1}\right)-k_{2} v\left(\xi_{1}\right)+k_{2}^{2} v\left(\xi_{1}\right)=0 .
$$

Hence, there exists $\delta>0$ such that $w(\xi)>k_{2} v(\xi)$ for $\xi \in\left(\xi_{1}, \xi_{1}+\delta\right)$ and $w(\xi)<k_{2} v(\xi)$ for $\xi \in\left(\xi_{1}-\delta, \xi_{1}\right)$. This implies that the direction field on $S_{2}$ is outward.

\section{UNSTABLE MANIFOLD OF PREDATOR-FREE EQUILIBRIUM}

Throughout this section, we assume that the conditions in Lemma 3.1 are satisfied; namely, $p>\mu$, $c>2 \sqrt{p-\mu}$ and $q<\varepsilon^{2}\left[c^{2} / 4-(p-\mu)\right]$. The predator-free equilibrium $(1,0)$ of the system (2.5)-(2.6) corresponds to the equilibrium $E_{0}=(1,0,0)$, which is also referred to as the predator-free equilibrium, of the dynamical system (3.1)-(3.3). The Jacobian matrix at $E_{0}$ is calculated as

$$
J_{0}=\left(\begin{array}{ccc}
-1 / c & -p / c & 0 \\
0 & c & -c \\
0 & (p-\mu) / c & 0
\end{array}\right) .
$$

In addition to a negative eigenvalue $-1 / c$, the matrix $J_{0}$ has two positive eigenvalues

$$
\lambda_{ \pm}=\frac{c \pm \sqrt{c^{2}-4(p-\mu)}}{2} .
$$

The eigenvectors associated with $\lambda_{ \pm}$are

$$
e_{ \pm}=\left(\begin{array}{c}
-p /\left(c \lambda_{ \pm}+1\right) \\
1 \\
1-\lambda_{ \pm} / c
\end{array}\right) .
$$

By [21, Theorem 3.2.1], the predator-free equilibrium $E_{0}$ possesses a smooth two-dimensional local invariant unstable manifold $W^{u}\left(E_{0}\right)$ tangent to the plane spanned by $e_{+}$and $e_{-}$. Note that

$$
1-\frac{\lambda_{ \pm}}{c}=\frac{1 \mp \sqrt{1-4(p-\mu) / c^{2}}}{2} \in\left(k_{1}, k_{2}\right)
$$

where $k_{1}$ and $k_{2}$ are given in (3.12). Hence, both vectors $e_{ \pm}$starting at the equilibrium $E_{0}=(1,0,0)$ point inward the pentahedron $P$. We then find a smooth curve $\gamma \in W^{u}\left(E_{0}\right) \cap P$ with two end points on $S_{1}$ and $S_{2}$, respectively. For each $i=1,2$, we denote $\gamma_{i}$ to be the point on $\gamma$ such that the trajectories starting from these points will remain in $P$ until touching $S_{i}$ at a finite time. Obviously, $\gamma_{1}$ and $\gamma_{2}$ are disjoint open subsets of $\gamma$. Since $\gamma$ is smooth and connected, there exists at least one point on $\gamma \backslash\left(\gamma_{1} \cup \gamma_{2}\right)$. The trajectory starting from this point will never touch $S_{1}$ or $S_{2}$, and hence stays in $P$ all the time. We summarize our argument in the following lemma. 
Lemma 4.1. Assume $p>\mu, c>2 \sqrt{p-\mu}$ and $q<\varepsilon^{2}\left[c^{2} / 4-(p-\mu)\right]$. There exists a trajectory $(u(\xi), v(\xi), w(\xi))$ of the dynamical system (3.1)-(3.3) such that

$$
\lim _{\xi \rightarrow-\infty}(u(\xi), v(\xi), w(\xi))=(1,0,0),
$$

and $(u(\xi), v(\xi), w(\xi)) \in P$ for all $\xi \in \mathbb{R}$.

\section{Heteroclinic orbit and Lyapunov function technique}

Let $(u(\xi), v(\xi), w(\xi))$ be the trajectory which connects to the predator-free equilibrium $E_{0}=(1,0,0)$ as $\xi \rightarrow-\infty$ and stays in the pentahedron $P$ for all $\xi \in \mathbb{R}$; see Lemma 4.1. We will prove in this section that this trajectory converges to the positive equilibrium $E_{1}=(U, V, V)$ as $\xi \rightarrow \infty$, where $(U, V)$ is the positive equilibrium of the original system (1.4)-(1.5). From (2.8), we calculate

$$
U=\frac{q+p \mu-\sqrt{(q+p \mu)^{2}-4 q \mu^{2}}}{2 q}, V=\frac{q-p \mu+\sqrt{(q+p \mu)^{2}-4 q \mu^{2}}}{2 q \mu} .
$$

It is easy to verify that $0<U<1$ and $k_{1}<1<k_{2}$. Moreover, in view of (2.8) and (3.6), we obtain

$$
c U+\varepsilon V+c V<\frac{U}{\varepsilon}+\frac{\mu V}{\varepsilon}=\frac{1}{\varepsilon} .
$$

Consequently, $(U, V, V) \in P$.

Lemma 5.1. Assume $p>\mu, c>2 \sqrt{p-\mu}$ and $q \leq Q$ with

$$
Q:=\min \left\{\frac{\varepsilon^{4} c^{2}(p-\mu)}{2 \mu^{2}}, \frac{2 \varepsilon^{4} c^{2}}{\mu}, \frac{\varepsilon^{2}\left[c^{2}-4(p-\mu)\right]}{5}\right\}
$$

and

$$
\varepsilon:=\min \left\{\frac{1}{2 c}, \frac{-c+\sqrt{c^{2}+4 \mu}}{4}, \frac{\mu^{3 / 4}}{c\left[4 / c^{2}+(\mu-1)^{2} /(4 \mu)\right]^{1 / 4}}\right\} .
$$

Let $(u(\xi), v(\xi), w(\xi))$ be the trajectory given in Lemma 4.1 and $(U, V, V)$ be the positive equilibrium calculated in (2.8). We have

$$
\lim _{\xi \rightarrow \infty}(u(\xi), v(\xi), w(\xi))=(U, V, V) .
$$

Proof. For any $(u, v, w) \in P$, we construct a Lyapunov function as

$$
L(u, v, w):=c\left(u-U \ln u+w-V \frac{w}{v}-V \ln v\right)+\frac{\kappa}{2 c}\left[\left(c u+\frac{\mu}{c} v+c w\right)-\left(c U+\frac{\mu}{c} V+c V\right)\right]^{2},
$$

where $\kappa>0$ is a positive constant to be determined later. Restricting the Lyapunov function on the trajectory $(u(\xi), v(\xi), w(\xi))$ and taking derivative with respect to $\xi$ yield

$$
\begin{aligned}
\frac{d}{d \xi} L(u(\xi), v(\xi), w(\xi))= & c\left[\left(1-\frac{U}{u(\xi)}\right) u^{\prime}(\xi)+\left(1-\frac{V}{v(\xi)}\right) w^{\prime}(\xi)+\left(\frac{V w}{v(\xi)^{2}}-\frac{V}{v(\xi)}\right) v^{\prime}(\xi)\right] \\
& +\frac{\kappa}{c}\left[c(u(\xi)-U)+\frac{\mu}{c}(v(\xi)-V)+c(w(\xi)-V)\right]\left[c u^{\prime}(\xi)+\frac{\mu}{c} v^{\prime}(\xi)+c w^{\prime}(\xi)\right] .
\end{aligned}
$$

For simplicity, we will drop the dependence on $\xi$ and write $u(\xi), v(\xi)$ and $w(\xi)$ as $u, v$ and $w$, respectively.. It then follows from (3.1)-(3.3) that

$$
\begin{aligned}
L^{\prime}= & {\left[\frac{u-U}{u}\left(1-u-p u v-q u v^{2}\right)+\frac{v-V}{v}\left(p u v+q u v^{2}-\mu v\right)+\frac{V(w-v)}{v^{2}} c^{2}(v-w)\right] } \\
& +\kappa\left[(u-U)+\frac{\mu}{c^{2}}(v-V)+(w-V)\right](1-u-\mu w) .
\end{aligned}
$$


Denote $\bar{u}=u-U, \bar{v}=v-V$ and $\bar{w}=w-V$. It is readily seen from (2.8) that

$$
\begin{aligned}
\frac{u-U}{u}\left(1-u-p u v-q u v^{2}\right) & =-\frac{1}{u U} \bar{u}^{2}-p \bar{u} \bar{v}-q \bar{u} \bar{v}^{2}-2 q V \bar{u} \bar{v}, \\
\frac{v-V}{v}\left(p u v+q u v^{2}-\mu v\right) & =p \bar{u} \bar{v}+q \bar{u} \bar{v}^{2}+q V \bar{u} \bar{v}+q U \bar{v}^{2}, \\
\frac{V(w-v)}{v^{2}} c^{2}(v-w) & =-\frac{c^{2} V}{v^{2}}\left(\bar{v}^{2}-2 \bar{v} \bar{w}+\bar{w}^{2}\right),
\end{aligned}
$$

and

$$
\left[(u-U)+\frac{\mu}{c^{2}}(v-V)+(w-V)\right](1-u-\mu w)=-\bar{u}^{2}-\frac{\mu}{c^{2}} \bar{u} \bar{v}-(\mu+1) \bar{u} \bar{w}-\frac{\mu^{2}}{c^{2}} \bar{v} \bar{w}-\mu \bar{w}^{2} .
$$

Note that $U \leq 1, u \leq 1$ and $v \leq 1 / \varepsilon^{2}$. We obtain from the above equations that

$$
\begin{aligned}
L^{\prime} \leq & {\left[-\bar{u}^{2}-q V \bar{u} \bar{v}+q U \bar{v}^{2}-\varepsilon^{4} c^{2} V\left(\bar{v}^{2}-2 \bar{v} \bar{w}+\bar{w}^{2}\right)\right] } \\
& +\kappa\left[-\bar{u}^{2}-\frac{\mu}{c^{2}} \bar{u} \bar{v}-(\mu+1) \bar{u} \bar{w}-\frac{\mu^{2}}{c^{2}} \bar{v} \bar{w}-\mu \bar{w}^{2}\right] .
\end{aligned}
$$

Now, we choose $\kappa=2 \varepsilon^{4} c^{4} V / \mu^{2}$ to eliminate $\bar{v} \bar{w}$ on the right-hand side of the above inequality. It follows that

$$
L^{\prime} \leq-(1+\kappa) \bar{u}^{2}-\left(q V+\frac{\kappa \mu}{c^{2}}\right) \bar{u} \bar{v}-\left(\frac{\kappa \mu^{2}}{2 c^{2}}-q U\right) \bar{v}^{2}-\kappa(\mu+1) \bar{u} \bar{w}-\left(\frac{\kappa \mu^{2}}{2 c^{2}}+\kappa \mu\right) \bar{w}^{2} \leq 0,
$$

provided $q<\varepsilon^{4} c^{2} V / U=\kappa \mu^{2} /\left(2 c^{2} U\right)$ and

$$
\frac{\left(q V+\kappa \mu / c^{2}\right)^{2}}{2 \kappa \mu^{2} / c^{2}-4 q U}+\frac{\kappa^{2}(\mu+1)^{2}}{2 \kappa \mu^{2} / c^{2}+4 \kappa \mu} \leq 1+\kappa .
$$

Note from (2.8) that $\mu V+U=1 \geq p U / \mu$. Especially, $V / U \geq(p-\mu) / \mu^{2}$. For any $q \leq Q$, we have $q V \leq \kappa \mu / c^{2}$ and $q U \leq \kappa \mu^{2} /\left(4 c^{2}\right)$. Consequently,

$$
\frac{\left(q V+\kappa \mu / c^{2}\right)^{2}}{2 \kappa \mu^{2} / c^{2}-4 q U}+\frac{\kappa^{2}(\mu+1)^{2}}{2 \kappa \mu^{2} / c^{2}+4 \kappa \mu}-\kappa \leq \frac{4 \kappa}{c^{2}}+\frac{\kappa(\mu+1)^{2}}{2 \mu^{2} / c^{2}+4 \mu}-\kappa \leq \frac{2 \varepsilon^{4} c^{4}}{\mu^{3}}\left[\frac{4}{c^{2}}+\frac{(\mu-1)^{2}}{4 \mu}\right],
$$

where we have made use of $\kappa=2 \varepsilon^{4} c^{4} V / \mu^{2}$ and $V \leq 1 / \mu$ in the last inequality. By the choice of $\varepsilon$ in (5.2), the right-hand side of the above inequality is no more than 1 . Hence, (5.4) is satisfied and $L^{\prime} \leq 0$ for all $q \leq Q$.

We claim that

$$
\lim _{\xi \rightarrow \infty} L(u(\xi), v(\xi), w(\xi))>-\infty .
$$

Assume to the contrary that $L(u(\xi), v(\xi), w(\xi)) \rightarrow-\infty$ as $\xi \rightarrow \infty$. It follows from the definition of $L$ in (5.3) that $v(\xi) \rightarrow 0$ as $\xi \rightarrow \infty$. For any small $\varepsilon_{0}>0$, there exists $\xi_{0} \in \mathbb{R}$ such that $p u(\xi) v(\xi)+$ $q u(\xi) v(\xi)^{2}<\varepsilon_{0}$ for all $\xi>\xi_{0}$. In view of (3.1), we have $c u^{\prime}>1-u-\varepsilon_{0}$ for all $\xi>\xi_{0}$. By comparison principle, we obtain $\liminf _{\xi \rightarrow \infty} u(\xi) \geq 1-\varepsilon_{0}$. Since $\varepsilon_{0}>0$ is arbitrary, letting $\varepsilon_{0} \rightarrow 0^{+}$ gives $\liminf \operatorname{in}_{\xi \rightarrow \infty} u(\xi) \geq 1$. This together with $u(\xi) \leq 1$ implies that $\lim _{\xi \rightarrow \infty} u(\xi)=1$. Note that $p>\mu$ and $v(\xi) \geq 0$. There exists $\xi_{1} \in \mathbb{R}$ such that $p u(\xi) v(\xi)-\mu v(\xi) \geq 0$ for all $\xi \geq \xi_{1}$. On account of (3.3), we obtain $c w^{\prime}(\xi) \geq 0$ for all $\xi \geq \xi_{1}$. Let $w_{\infty}=\lim _{\xi \rightarrow \infty} w(\xi) \geq 0$. If $w_{\infty}>0$, then there exists $\xi_{2} \in \mathbb{R}$ such that $v(\xi)-w(\xi)<-w_{\infty} / 2$ for all $\xi>\xi_{2}$. It follows from (3.2) that $v^{\prime}(\xi)<-c w_{\infty} / 2$ for $\xi>\xi_{2}$, which contradicts to $\lim _{\xi \rightarrow \infty} v(\xi)=0$. Hence, $w_{\infty}=\lim _{\xi \rightarrow \infty} w(\xi)=0$. Since $w(\xi) \geq 0$ and $c w^{\prime}(\xi) \geq 0$ for all $\xi \geq \xi_{1}$, we obtain $w(\xi)=0$ for all $\xi \geq \xi_{1}$. Choose $\xi_{3} \leq \xi_{1}$ such that $w(\xi)=0$ for all $\xi \geq \xi_{3}$ and $w(\xi)>0$ if $\xi$ is smaller than and close to $\xi_{3}$. In view of $(3.2)$ and $\lim _{\xi \rightarrow \infty} v(\xi)=0$, we obtain 
$v(\xi)=0$ for all $\xi \geq \xi_{3}$. Since the line $v=w=0$ is negatively invariant for the system (3.1)-(3.3), we have $v(\xi)=w(\xi)=0$ for all $\xi \in \mathbb{R}$, a contradiction.

The above argument implies that

$$
L_{\infty}:=\lim _{\xi \rightarrow \infty} L(u(\xi), v(\xi), w(\xi))>-\infty .
$$

Now, we apply LaSalle invariance principle to show that the trajectory $(u(\xi), v(\xi), w(\xi))$ converges to the positive equilibrium $(U, V, V)$ as $\xi \rightarrow \infty$. Let $\left(\tilde{u}_{0}, \tilde{v}_{0}, \tilde{w}_{0}\right)$ be any point in the omega limit set of the trajectory $(u(\xi), v(\xi), w(\xi))$; namely, there exists a subsequence $\xi_{1}<\xi_{2}<\cdots<\xi_{n} \rightarrow \infty$ such that $\left(u\left(\xi_{n}\right), v\left(\xi_{n}\right), w\left(\xi_{n}\right)\right) \rightarrow\left(\tilde{u}_{0}, \tilde{v}_{0}, \tilde{w}_{0}\right)$ as $n \rightarrow \infty$. Let $(\tilde{u}(\xi), \tilde{v}(\xi), \tilde{w}(\xi))$ be the solution of (3.1)-(3.3) with $(\tilde{u}(0), \tilde{v}(0), \tilde{w}(0))=\left(\tilde{u}_{0}, \tilde{v}_{0}, \tilde{w}_{0}\right)$. For any $\xi \in \mathbb{R}$, we then have $\left(u\left(\xi_{n}+\xi\right), v\left(\xi_{n}+\xi\right), w\left(\xi_{n}+\xi\right)\right) \rightarrow$ $(\tilde{u}(\xi), \tilde{v}(\xi), \tilde{w}(\xi)) \in P$ as $n \rightarrow \infty$. Especially, we obtain

$$
L(\tilde{u}(\xi), \tilde{v}(\xi), \tilde{w}(\xi))=\lim _{n \rightarrow \infty}\left(u\left(\xi_{n}+\xi\right), v\left(\xi_{n}+\xi\right), w\left(\xi_{n}+\xi\right)\right)=L_{\infty},
$$

and thus

$$
\frac{d}{d \xi} L(\tilde{u}(\xi), \tilde{v}(\xi), \tilde{w}(\xi))=0
$$

From the proof of $L^{\prime} \leq 0$, we conclude that $(\tilde{u}(\xi), \tilde{v}(\xi), \tilde{w}(\xi))=(U, V, V)$ for all $\xi \in \mathbb{R}$. Therefore, the omega limit set of the trajectory $(u(\xi), v(\xi), w(\xi))$ is a singleton $(U, V, V)$, which implies that $(u(\xi), v(\xi), w(\xi)) \rightarrow(U, V, V)$ as $\xi \rightarrow \infty$. This completes the proof.

\section{Proof of Theorem 2.1}

The existence result follows from a sequence of Lemma 3.1, Lemma 4.1 and Lemma 5.1. To establish the nonexistence result, we assume to the contrary that $(u(\xi), v(\xi))$ is a positive solution to the boundary value problem (2.5)-(2.7). Define $w(\xi)=v(\xi)-v^{\prime}(\xi) / c$. Then, $(u(\xi), v(\xi), w(\xi))$ is a solution to the dynamical system (3.1)-(3.3) such that $v(\xi)>0$ and $(u(\xi), v(\xi), w(\xi)) \rightarrow(1,0,0)$ as $\xi \rightarrow-\infty$. Recall that the Jacobian matrix $J_{0}$ about the predator-free equilibrium $E_{0}=(1,0,0)$ has three eigenvalues $-1 / c$ and $\lambda_{ \pm}$; see (4.1) and (4.2). Since $c<2 \sqrt{p-\mu}$, the eigenvalues $\lambda_{ \pm}$are complex with positive real parts and nonzero imaginary parts. There exist two (complex conjugate) constants $c_{ \pm}$such that

$$
\left(\begin{array}{c}
u(\xi) \\
v(\xi) \\
w(\xi)
\end{array}\right)=\left(\begin{array}{l}
1 \\
0 \\
0
\end{array}\right)+c_{+} e^{\lambda_{+} \xi} e_{+}+c_{-} e^{\lambda_{-}} e_{-}+o\left(e^{\operatorname{Re} \lambda_{+} \xi}\right),
$$

as $\xi \rightarrow-\infty$, where $e_{ \pm}$are the eigenvectors associated with the eigenvalues $\lambda_{ \pm}$; see (4.3). Especially, $v(\xi)$ oscillates around 0 as $\xi \rightarrow-\infty$. This contradicts to the assumption that $v(\xi)>0$ for all $\xi \in \mathbb{R}$. The proof is complete.

\section{Discussion}

In this paper, we have established an existence theory of traveling wave solutions for a diffusive predator-prey system with cooperative hunting. The main challenge lies in the fact that the function of cooperative predation is not dominated by a linear function; namely, the sublinearity condition is not satisfied. We first transform the traveling wave equations to a dynamical system. By using an extension of the approach introduced in $[12,13]$, we construct a suitable pentahedron, and find inside this pentahedron a trajectory connecting the predator-free equilibrium at one end. Lyapunov function technique and LaSalle invariance principle are applied to prove that this trajectory connects the positive 
equilibrium at the other end. Our result indicates that there exists a critical wave speed $c^{*}$ such that no positive traveling wave solution exists when $c<c^{*}$. On the other hand, if $c>c^{*}$, then a positive traveling wave solution exists for any sufficiently small cooperative predation rate $q$.

There are some open problems related to traveling waves in cooperative predation. (1) Strong cooperative predation. In our analysis, we need a technical assumption $q<Q$, where $Q$ is a positive constant depending on the model parameters and the wave speed. It is conjectured that when $q \geq Q$, there still exists a positive traveling wave solution if $c>c^{*}$. (2) Prey diffusion. In our model, we simply assume that the diffusion rate of the prey is zero. It is reasonable to expect that a similar result holds when the prey has a positive diffusion rate. In this case, one needs to investigate a four-dimensional dynamical system and construct a suitable polychoron (i.e., 4-polytope). We leave this problem as future work. (3) Critical case. We have proved the existence of traveling wave solution when $c>c^{*}$ and the non-existence of traveling wave solution when $c<c^{*}$. We conjecture that the traveling wave solution exists for the critical case $c=c^{*}$. (4) Bistable equilibria. We assume that the basic reproduction number $R_{0}$ is greater than one and hence there exists a unique positive equilibrium for the diffusion-free system. However, if $R_{0}<1$, the diffusion-free system may have two positive equilibria with one unstable and the other locally asymptotically stable. It is interesting to study the traveling wave solution connecting the unstable positive equilibrium to the stable positive equilibrium. One may also want to investigate the traveling wave solution connecting the unstable positive equilibrium to the (stable) predation-free equilibrium. (5) General prey growth. In our model, we assume that the prey growth function is linear. It is natural to ask whether one could extend our study to the general case when the growth rate of the prey is a general function such as the logistic (quadratic) function or the Allee effect (cubic) function.

\section{ACKNOWLEDGMENT}

We would like to thank two anonymous referees for careful reading and helpful suggestions which led to an improvement to our original manuscript.

\section{REFERENCES}

[1] M. Alves and F.M. Hilker, Hunting cooperation and Allee effects in predators, J. Theo. Biol. 419 (2017), 13-22.

[2] G. Beauchamp, Social Predation: How Group Living Benefits Predators and Prey, Academic Press, 2014.

[3] J. Bednarz, Cooperative hunting Harris' hawks (Parabuteo unicinctus), Science 239 (1988), 1525-1527.

[4] R. Chisholm and R. Firtel, Insights into morphogenesis from a simple developmental system, Nat. Rev. Mol. Cell. Biol. 5 (2004), 531-541.

[5] W. Ding and W. Huang, Traveling wave solutions for some classes of diffusive predator-prey models, J. Dynam. Differential Equations 28 (2016), 1293-1308.

[6] S. R. Dunbar, Traveling wave solutions of diffusive Lotka-Volterra equations, J. Math. Biol. 17 (1983), 11-32.

[7] _ Traveling wave solutions of diffusive Lotka-Volterra equations: A heteroclinic connection in $R^{4}$, Trans. Amer. Math. Soc. 286 (1984), 557-594.

[8] Y. Hosono and B. Ilyas, Existence of traveling waves with any positive speed for a diffusive epidemic model, Nonlinear World 1 (1994), 277-290.

[9] _ Traveling waves for a simple diffusive epidemic model, Math. Models Methods Appl. Sci. 5 (1995), 935-966.

[10] C.-H. Hsu, C.-R. Yang, T.-H. Yang, and T.-S. Yang, Existence of traveling wave solutions for diffusive predator-prey type systems, J. Differential Equations 252 (2012), 3040-3075.

[11] J. Huang, G. Lu, and S. Ruan, Existence of traveling wave solutions in a diffusive predator-prey model, J. Math. Biol. 46 (2003), 132-152.

[12] W. Huang, Traveling wave solutions for a class of predator-prey systems, J. Dynam. Differential Equations 24 (2012), 633-644. 
[13] _ _ A geometric approach in the study of traveling waves for some classes of non-monotone reaction-diffusion systems, J. Differential Equations 260 (2016), 2190-2224.

[14] S. R.-J. Jang, W. Zhang, and V. Larriva, Cooperative hunting in a predator-prey system with Allee effects in the prey, Nat. Resour. Model. 31 (2018), e12194.

[15] S. I. Li and M. D. Purugganan, The cooperative amoeba: Dictyostelium as a model for social evolution, Trends Genet. 27 (2011), 48-54.

[16] W.-T. Li and S.-L. Wu, Traveling waves in a diffusive predator-prey model with holling type-III functional response, Chaos Solitons Fractals 37 (2008), 476-486.

[17] A. Lotka, Elements of Physical Biology, Williams and Wilkins, Baltimore, 1925.

[18] D. R. MacNulty, A. Tallian, D. R. Stahler, and D. W. Smith, Influence of group size on the success of wolves hunting bison, PLOS ONE 9 (2014), e112884.

[19] C. Packer and L. Ruttan, The evolution of cooperative hunting, Am. Nat. 132 (1988), 159-198.

[20] V. Volterra, Fluctuations in the abundance of a species considered mathematically, Nature 118 (1926), 538-560.

[21] S. Wiggins, Introduction to Applied Nonlinear Dynamical Systems and Chaos, Texts in Applied Mathematics, vol. 5, Springer, New York, 1990.

[22] T. Zhang, W. Wang, and K. Wang, Minimal wave speed for a class of non-cooperative diffusion-reaction system, J. Differential Equations 260 (2016), 2763-2791.

Department of Mathematics, University of Louisiana at Lafayette, Lafayette, LA 70503, USA.

E-mail address: srijana.ghimire1@louisiana.edu

Corresponding author, Department of Mathematics, University of Louisiana at lafayette, Lafayette, LA 70503, USA.

E-mail address: xswang@louisiana.edu 